09

\title{
Динамика спектров квантово-каскадных лазеров, генерирующих частотные гребенки в длинноволновом инфракрасном диапазоне
}

\author{
() В.В. Дюделев, ${ }^{1}$ Д.А. Михайлов, ${ }^{1}$ А.В. Бабичев, ${ }^{1}$ С.Н. Лосев, ${ }^{1}$ Е.А. Когновицкая, ${ }^{1,2}$ А.В. Лютецкий, ${ }^{1}$ \\ С.О. Слипченко, ${ }^{1}$ Н.А. Пихтин, ${ }^{1}$ А.Г. Гладышев, ${ }^{3}$ Д.В. Денисов, ${ }^{2}$ И.И. Новиков, ${ }^{1,3,4}$ Л.Я. Карачинский, ${ }^{1,3,4}$ \\ В.И. Кучинский, ${ }^{1}$ А.Ю. Егоров, ${ }^{4}$ Г.С. Соколовский ${ }^{1, \uparrow}$
}

${ }^{1}$ Физико-технический институт им. А.Ф. Иофрфе РАН, 194021 Санкт-Петербург, Россия

${ }^{2}$ СПбГЭТУ „ЛЭТИ“, 197376 Санкт-Петербург, Россия

${ }^{3} \mathrm{OOO} \mathrm{„Коннектор} \mathrm{Оптикс“,}$

194292 Санкт-Петербург, Россия

${ }^{4}$ Университет ИТМО,

197101 Санкт-Петербург, Россия

『e-mail: gs@mail.ioffe.ru

Поступило в редакцию 10 марта 2020 г.

В окончательной редакции 10 марта 2020 г.

Принято к публикации 11 марта 2020 г.

Исследованы спектральные и динамические характеристики квантово-каскадных лазеров, излучающих в длинноволновом инфракрасном диапазоне. Показано, что лазеры с коротким резонатором $(\sim 1 \mathrm{~mm})$ позволяют получить частотные гребенки в очень широком спектральном диапазоне. Проведено исследование динамики спектров в режиме генерации частотных гребенок. Показано изменение интенсивности продольных мод лазерной генерации в течение импульса накачки. При этом наблюдалась одновременная генерация всех продольных мод частотной гребенки в течение участка импульса накачки с постоянной амплитудой.

Ключевые слова: квантово-каскадный лазер, частотные гребенки, ИК-диапазон.

DOI: $10.21883 / J T F .2020 .08 .49544 .78-20$

\section{Введение}

Интенсивные исследования по созданию квантовокаскадных лазеров (ККЛ) для длинноволнового инфракрасного диапазона связаны с наличием в нем интенсивных линий поглощения многих химических соединений, а также с использованием второго окна прозрачности атмосферы. Поэтому среди возможных применений таких ККЛ можно выделить газоанализ, мониторинг состояния окружающей среды, неразрушающий дистанционный контроль, а также беспроводную оптическую связь.

В связи с этим актуальной задачей является разработка систем спектрального экспресс-анализа. Наиболее перспективным подходом являются системы, основанные на интерференции двух частотных гребенок с небольшим отличием в межмодовом расстоянии [1]. Важной особенностью метода является одновременная генерация всех мод частотной гребенки для получения расшифровываемой интерференционной картины на фотоприемнике. Следует отметить, что применение частотных гребенок в области спектроскопии атомных переходов [2,3] было отмечено Нобелевской премией по физике 2005 года. В настоящее время ведутся активные исследования по получению частотных гребенок от ККЛ длинноволнового инфракрасного диапазона [4-9] с применением методов активной синхронизации мод [4-7], а также за счет возникновения самопульсаций в ККЛ $[8,9]$.
Настоящая работа посвящена исследованию недостаточно изученного вопроса динамики спектров ККЛ при генерации частотных гребенок.

\section{Экспериментальные образцы}

В настоящей работе исследуется генерация частотных гребенок ККЛ, излучающих вблизи $8 \mu \mathrm{m}$, а также их динамика. Исследуемые ККЛ были изготовлены на основе гетероструктуры, подробно описанной в работе [10]. С использованием данной гетероструктуры ранее была получена одночастотная генерация ККЛ с арочным резонатором [11], мощные ККЛ [12,13], генерирующие более $1 \mathrm{~W}$ выходной мощности, а также продемонстрированы двухчастотная [14] и высоко-температурная генерации [15]. Как было показано ранее [9], на основе данной гетероструктуры можно получить генерацию частотных гребенок с шириной частотного спектра $\sim 2 \mathrm{THz}$. Методами постростовой обработки из гетероструктуры были изготовлены полосковые ККЛ с шириной полоска $\sim 17 \mu \mathrm{m}$. На гетероструктуре были вытравлены неглубокие мезы (до активной области), формирующие гребневый контакт. Далее из гетероструктуры выкалывались ККЛ с длинами резонатора $\sim 1 \mathrm{~mm}$. ККЛ монтировались на первичный теплоотвод гетероструктурой вверх в связи с выбранной технологией формирования гребневого 
контакта. Анти/высокоотражающие покрытия на выходные грани исследуемых экспериментальных образцов не наносились.

\section{Эксперимент}

ККЛ исследовались в импульсном режиме генерации. Длительность токовых импульсов на половине от максимальной амплитуды составляла $\sim 75 \mathrm{~ns}$ с частотой повторения $48 \mathrm{kHz}$. Ватт-амперная характеристика измерялась при помощи измерителя мощности Thorlabs PM100 с термоэлектрической головкой S401C. Типичная ватт-амперная характеристика для излучения, собранного с одного выходного зеркала, приведена на рис. 1. Максимальная выходная пиковая мощность с одного зеркала превышала $600 \mathrm{~mW}$, что при нанесении на выходные грани ККЛ анти/высокоотражающих покрытий позволило бы получить свыше одного вата выходной оптической мощности.

Исследования усредненных спектров ККЛ проводились при помощи монохроматора МДР-23 с дифракционной решеткой $75 \mathrm{~mm}^{-1}$. Излучение регистрировалось быстродействующим фотоприемником Vigo Systems PVI-4TE-10.6 с полосой пропускания $1 \mathrm{GHz}$. На рис. 2 приведены усредненные спектры генерации для ККЛ с длиной резонатора 1 и $1.2 \mathrm{~mm}$ при токе накачки $10 \mathrm{~A}$.

Проведенные спектральные измерения показали, что исследуемые образцы ККЛ генерируют широкие частотные гребенки. Причем увеличение длины резонатора приводит к постепенному сужению спектра и количества участвующих в генерации продольных мод. Так, для лазера с длиной резонатора $1 \mathrm{~mm}$ ширина спектра частотной гребенки превысила $2.2 \mathrm{THz}$ и количество

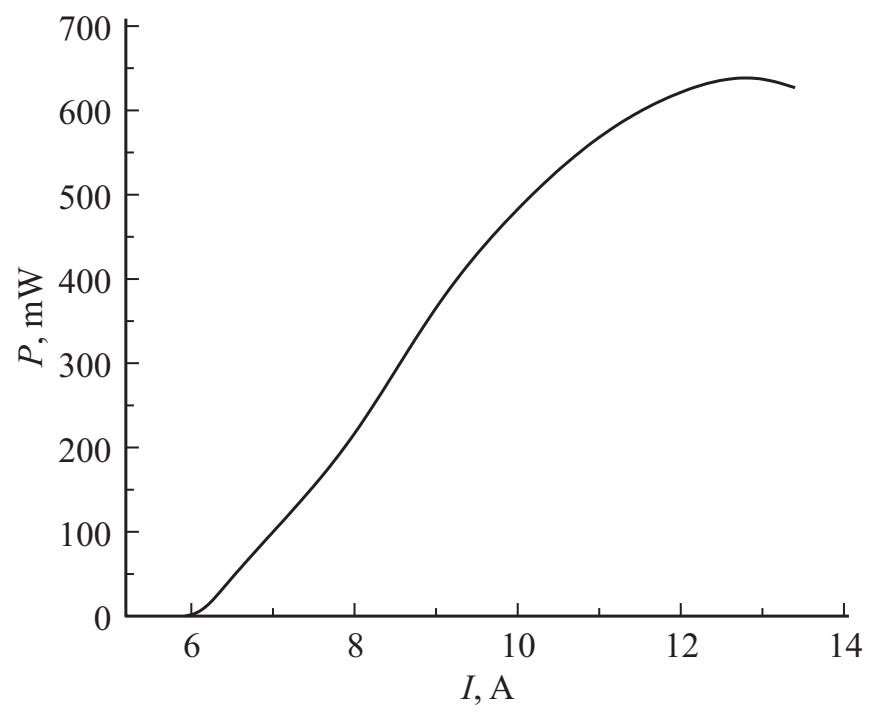

Рис. 1. Типичная ватт-амперная характеристика ККЛ с шириной полоска $17 \mu \mathrm{m}$ и длиной резонатора $\sim 1 \mathrm{~mm}$ с одного зеркала при комнатной температуре.
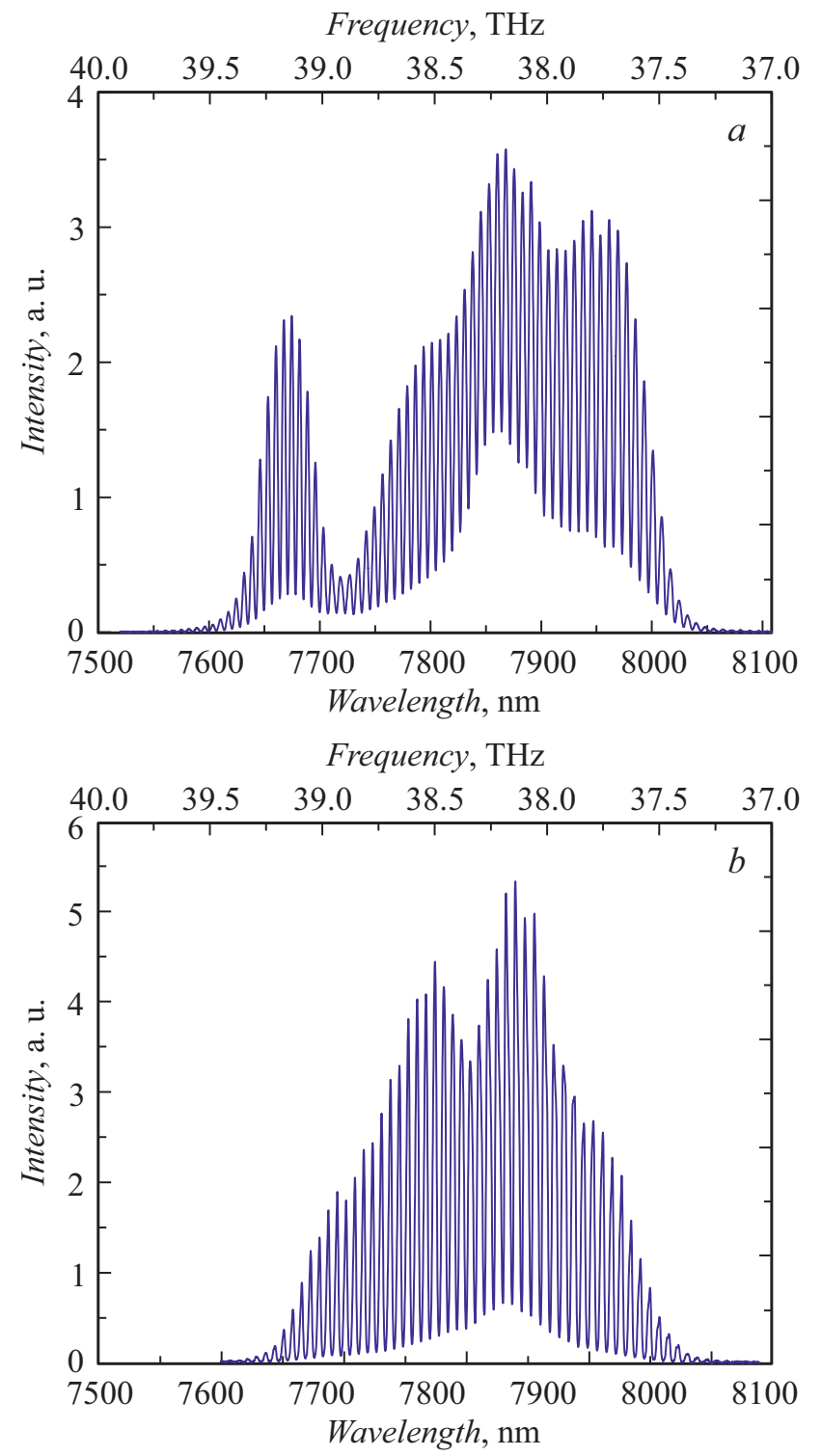

Рис. 2. Усредненный спектр генерации ККЛ при амплитуде тока накачки $10 \mathrm{~A}: a-\mathrm{c}$ длиной резонатора $1 \mathrm{~mm} ; b-\mathrm{c}$ длиной резонатора $1.2 \mathrm{~mm}$.

участвующих в генерации мод превышает 60. В то же время при увеличении длины резонатора на $200 \mu \mathrm{m}$ заметно сужение спектра частотной гребенки до $1.8 \mathrm{THz}$ при количестве мод, участвующих в генерации, менее 50.

Далее нами были проведены исследования спектров генерации с временным разрешением, позволяющие исследовать динамику продольных мод в ККЛ с резонатором Фабри-Перо при генерации частотных гребенок. Методика эксперимента заключалась в следующем. Излучение лазера собиралось линзой из фторида бария и пропускалось через монохроматор МДР-23, за выходной щелью которого располагался быстродействующий фотоприемник Vigo Systems PVI-4TE-10.6, подключенный к осциллографу Agilent с полосой пропускания $1 \mathrm{GHz}$. При помощи осциллографа последовательно на каждом спек- 


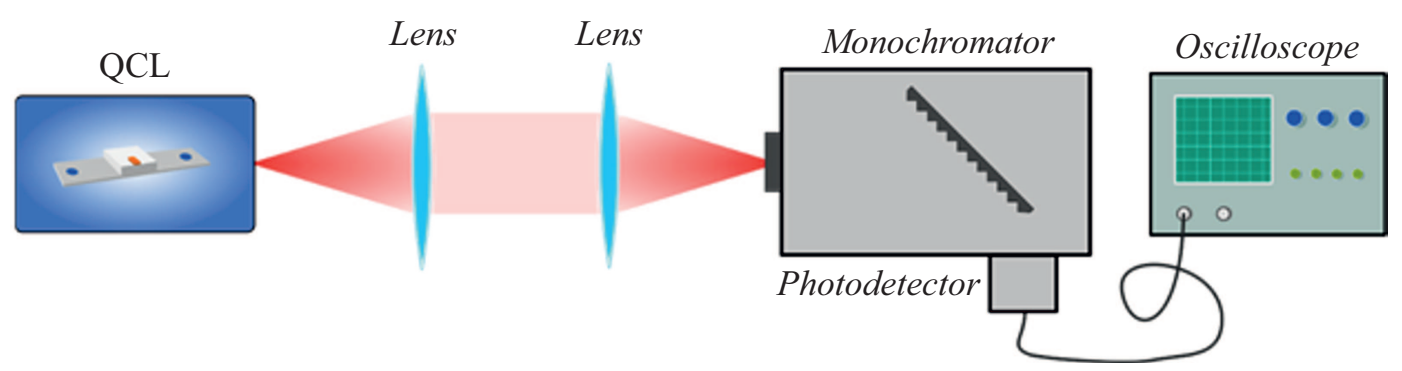

Рис. 3. Схема установки для измерения спектров ККЛ с временным разрешением.
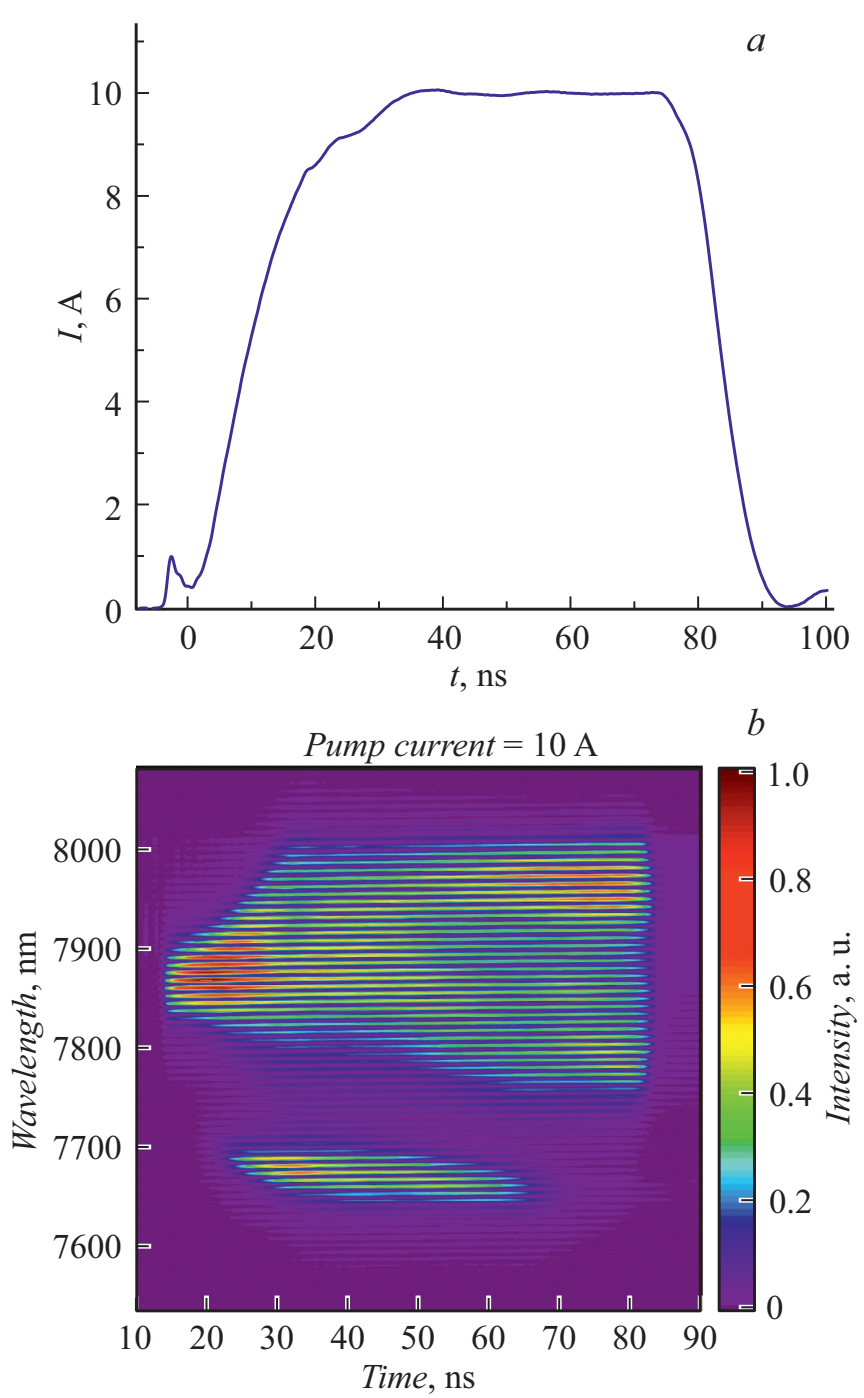

Pис. 4. $a-$ - форма импульса накачки с амплитудой тока $10 \mathrm{~A} ; b-$ результаты измерений динамики спектров ККЛ, генерирующего частотную гребенку.

тральном шаге регистрировались осциллограммы, описывающие распределение интенсивности в спектральной области, определяемой разрешением монохроматора. На основании полученных данных строилась зависимость распределения интенсивности выходного излучения от длины волны и времени. Схема установки представлена на рис. 3.

На рис. 4, $b$ представлены результаты измерений спектров ККЛ с временным разрешением при токе накачки 10 А. Форма импульса накачки представлена на рис. 4, $a$.

Из данных, представленных на рис. 4, видно, что происходит изменение интенсивности продольных мод лазерной генерации в процессе накачки. Начало генерации ККЛ происходит на спектральной полосе с максимумом вблизи $7850 \mathrm{~nm}$, затем при достижении импульсом накачки постоянной амплитуды, наиболее интенсивной становится коротковолновая область спектра генерации вблизи $7670 \mathrm{~nm}$, а ближе к заднему фронту импульса накачки наиболее интенсивной становится наиболее длинноволновая спектральная полоса с максимумом вблизи $8 \mu \mathrm{m}$. При этом следует отметить, что наблюдается одновременная генерация всех мод частотной гребенки в течение всего участка импульса накачки с постоянной амплитудой.

\section{Выводы}

Проведены исследования динамики спектров ККЛ длинноволнового инфракрасного диапазона при генерации широкополосных частотных гребенок с шириной полосы генерации более $2 \mathrm{THz}$. Спектральные измерения с временным разрешением показали изменение интенсивности продольных мод лазерной генерации в течение импульса накачки. При этом наблюдается одновременная генерация всех продольных мод частотной гребенки в течение участка импульса накачки с постоянной амплитудой.

\section{Благодарности}

Работа выполнена при поддержке Министерства науки и высшего образования РФ (уникальный идентификатор проекта RFMEFI61619X0111).

\section{Конфоликт интересов}

Авторы заявляют, что у них нет конфликта интересов. 


\section{Список литературы}

[1] Coddington I., Newbury N., Swann W. // Optica. 2016. Vol. 3. P. 414-426.

[2] Reichert J., Niering M., Holzwarth R., Weitz M., Udem Th., Hänsch T.W. // Phys. Rev. Lett. 2000. Vol. 84. P. 2264.

[3] Holzwarth R., Udem Th., Hänsch T.W., Knight J.C., Wadsworth W.J., Russell P.St. // Phys. Rev. Lett. 2000. Vol. 85. P. 3232.

[4] Faist J., Villares G., Scalari, Rösch M., Bonzon C., Hugi A., Beck M. // Nanophotonics. 2016. Vol. 5. P. 272.

[5] Hillbrand J., Andrews A.M., Detz H., Strasser G., Schwarzet B. // Nat. Phot. 2019. Vol. 13. P. 101.

[6] Capasso F., Gmachl C., Paiella R., Tredicucci A., Hutchinson A.L., Sivco D.L., Baillargeon J.N., Cho A.Y., Liu H.C. // IEEE J. Sel. Top. Quant. Electron. 2000. Vol. 6. P. 12929.

[7] Wang C.Y., Kuznetsova L., Gkortsas V.M., Diehl L., Kärtner F.X., Belkin A., Belyanin A., Li X., Ham D., Schneider H., Grant P., Song C.Y., Haffouz S., Wasilewski Z.R., Liu H.C., Capasso F. // Opt. Exp. 2009. Vol. 17. P. 145.

[8] Gordon A., Wang C.Y., Diehl L., Kärtner F.X., Belyanin A., Bour D., Corzine S., Höfler G., Liu H.C., Schneider H., Maier T., Troccoli M., Faist J., Capasso F. // Phys. Rev. A. 2008. Vol. 77. P. 053804.

[9] Dudelev V.V., Mikhailov D.A., Babichev A.V., Losev S.N., Chistyakov D.V., Kognovitskaya E.A., Avrov D.D., Slipchenko S.O., Lyutetskii A.V., Pikhtin N.A., Gladyshev A.G., Karachinsky L.Ya., Novikov I.I., Kuchinskii V.I., Egorov A.Yu., Sokolovskii G.S. // Tech. Phys. Lett. 2019. Vol. 45. P. 1027.

[10] Babichev A.V., Gladyshev A.G., Filimonov A.V., Nevedomskii V.N., Kurochkin A.S., Kolodeznyi E.S., Sokolovskii G.S., Bugrov V.E., Karachinsky L.Ya., Novikov I.I., Bousseksou A., Egorov A.Yu. // Tech. Phys. Lett. 2017. Vol. 43. P. 666.

[11] Babichev A.V., Gladyshev A.G., Kurochkin A.S., Dudelev V.V., Kolodeznyi E.S., Sokolovskii G.S., Bugrov V.E., Karachinsky L.Ya., Novikov I.I., Denisov D.V., Ionov A.S., Slipchenko S.O., Lyutetskii A.V., Pikhtin N.A., Egorov A.Yu. // Tech. Phys. Lett. 2018. Vol. 44. P. 398.

[12] Babichev A.V., Dudelev V.V., Gladyshev A.G., Mikhailov D.A., Kurochkin A.S., Kolodeznyi E.S., Bougrov V.E., Nevedomskiy V.N., Karachinsky L.Y., Novikov I.I., Denisov D.V., Ionov A.S., Slipchenko S.O., Lutetskiy A.V., Pikhtin N.A., Sokolovskii G.S., Egorov A.Y. // Tech. Phys. Lett. 2019. Vol. 45. P. 735.

[13] Dudelev V.V., Mikhailov D.A., Babichev A.V., Andreev A.D., Losev S.N., Kognovitskaya E.A., Bobretsova Yu.K., Slipchenko S.O., Pikhtin N.A., Gladyshev A.G., Denisov D.V., Novikov I.I., Karachinsky L.Ya., Kuchinskii V.I., Egorov A.Yu., Sokolovskii G.S. // Quant. Electron. 2020. Vol. 50. P. 141.

[14] Dudelev V.V., Losev S.N., Mylnikov V.Y., Babichev A.V., Kognovitskaya E.A., Slipchenko S.O., Lutetskii A.V., Pikhtin N.A., Gladyshev A.G., Karachinskii L.Ya., Novikov I.I., Egorov A.Yu., Kuchinskii V.I., Sokolovskii G.S. // Opt. Spectr. 2018. Vol. 125. P. 402.

[15] Dudelev V.V., Losev S.N., Myl'nikov V.Yu., Babichev A.V., Kognovitskaya E.A., Slipchenko S.O., Lyutetskii A.V., Pikhtin N.A., Gladyshev A.G., Karachinsky L.Ya., Novikov I.I., Egorov A.Yu., Kuchinskii V.I., Sokolovskii G.S. // Tech. Phys. 2018. Vol. 63. P. 1656. 